\title{
Study on Supply Chain Contracts with Asymmetric Information under Disruptions
}

\author{
Pin ZHUANG \\ College of Economics and Management, Nanjing University of Aeronautics and Astronautics, Nanjing \\ 210016, China
}

JyeChyi LU

School of Industrial and Systems Engineering, Georgia Institute of Technology, Atlanta, GA 30332-0205, USA

\begin{abstract}
This paper studies supply chain contract models with asymmetric information when the retailer's cost is disrupted. While the retailer faces a non-linear demand function, supply chain contract models under asymmetric information are proposed in a regular scenario. When the retailer's cost distribution is fluctuated by disruptions, the new adjusted policies as emergency strategies of supply chain under asymmetric information are obtained, which can still coordinate the supply chain under disruptions. Using numerical methods, the impact of the cost disruptions on decisions about optimal order quantity and system expected profit are analyzed.
\end{abstract}

KEYWORD: Supply Chain Contract; Disruption Management; Asymmetric Information

\section{INTRODUCTION}

Supply chain contracts are widely used as a coordination mechanism in supply chain management. Oliveira et al. [1] proposed a supply chain contract in electricity markets with multiple generators and retailers and considered several market structures. Seifert et al. [2] studied the priceonly contracts in a three-echelon supply chain and examines the impact of sub-supply chain coordination (sub-coordination).

In the absence of any disruption, with the above contracts, the supply chain can achieve channel coordination. However, various unexpected events such as September 11, the West Coast port lockout of 2002, hurricanes Katrina and Rita in 2005 disrupt the system and lead to huge losses. Supply chain disruption management is concerned with how to make optimal reacting decisions when system faces disruptions, so as to minimize the loss caused by disruptions. Guarnaschelli et al. [3] introduced a generalized model to provide automation to the disruption management function of Supply Chain Event Management (SCEM) systems. Schmitt ${ }^{[4]}$ investigated a multi-echelon system where disruptions can occur at any stage and evaluated multiple strategies for protecting customer service if a disruption should occur. Sawik [5] dealt with the optimal selection of supply portfolio in a make-toorder environment in the presence of supply chain disruption risks.

A critical assumption made in previous supply chain literatures is that the supplier has complete knowledge about the retailer's cost structure. Clearly, this assumption will seldom be fulfilled in practice. Caoa et al. [6] modeled the optimal wholesale contract design problem in a dual-channel supply chain under information asymmetry. Chu and Leon [7] studied the problems of coordinating serial and assembly inventory systems with private information where end-item demands are known over a finite horizon.

\section{SUPPLY CHAIN CONTRACTS WITH ASYMMETRIC INFORMATION}

\subsection{The basic supply chain contracts with asymmetric information}

Consider a two-echelon supply chain consisting of a single supplier and single retailer, both of whom are independent decision-makers seeking to maximize their individual profit. The retailer faces algebraic demand function $D(p)=(k p+b)^{-\alpha}$, where $k>0$, $b \neq 0$ and $\alpha>1$ are known parameters, $p$ is the retail price selling to the customer. Suppose the supplier's variable costs are $s$, the retailer's internal variable costs are $c$. The wholesale price is $w$, the retailer's order quantity is $Q$.

In general, $c$ is a stochastic and asymmetric variable, i.e., the retailer has private information about his cost construction, which the supplier dose 
not know. We assume $F(c)$ is prior distribution function of $c$, with continuous density function $f(c)$, and mean value of $\mu . F(c)$ is differentiable, strictly increasing and is defined on $[\underline{c}, \bar{c}]$, where $0 \leq \underline{c} \leq \bar{c} \leq \infty$. Let $F(0)=0$ and $\bar{F}(c)=1-F(c)$. All parameters except $c$ are common knowledge.

To provide a benchmark, we first investigate the centralized situation where one central decision maker seeks to maximize total system profits. The supply chain system's expected profit can be written as

$$
\Pi^{c}(p)=\int_{\underline{c}}^{\bar{c}}\left[(k p+b)^{-\alpha}(p-s-c)\right] f(c) d c .
$$

It is easy to see that $\Pi^{c}(p)$ is strictly concave over $p$, so there must be a unique optimal point $p$ to maximize the supply chain system profit. By the first-order condition $\partial \Pi^{c}(p) / \partial p=0$, we have the optimal retail price $p^{c}=[\alpha(s+\mu)+b / k] /(\alpha-1)$, and the optimal order quantity $Q^{c}=(\alpha-1)^{\alpha} /[\alpha k(s+\mu+b / k)]^{\alpha}$. Then the supply chain system's expected profit is $\Pi^{c}=(\alpha-1)^{\alpha-1} /\left[(\alpha k)^{\alpha}(s+\mu+b / k)^{\alpha-1}\right]$.

\subsection{The supply chain contracts under disruptions}

Now we consider the situation with asymmetric information under disruptions. We assume that there are only retailer's cost disruptions and other settings are unchanged. The situation is as follows, after the supplier's production plan is made and before the selling season is coming, unforeseeable events take place, and it makes the retailer's cost disruption, i.e., the cost distribution changes from $F$ to $G$ (its density function is assumed to be $g$ ). Same as the distribution function $F$, we assume the distribution function of demand $G$ is differentiable and strictly increasing and $G(0)=0$. Let $\bar{G}(c)=1-G(c)$ and denote the mean value as $\tilde{\mu}$. Under the disruptions, we assume the optimal emergency production quantity is $\tilde{Q}$.

When an original plan is revised, there will be some deviation cost associated with the adjustment. By considering the deviation cost, the supply chain system's expected profit function under disruptions can be written as:

$\tilde{\Pi}^{c}(\tilde{p})=E\left[\tilde{Q}(\tilde{p}-s-c)-\varepsilon_{1}\left(\tilde{Q}-Q^{c}\right)^{+}-\varepsilon_{2}\left(Q^{c}-\tilde{Q}\right)^{+}\right]$,

where the parameters $\varepsilon_{1}$ and $\varepsilon_{2}$ are the marginal extra costs associated with the adjustment the production plan from the original plan respectively, and $(x)^{+}=\max (0, x)$. More precisely, $\varepsilon_{1}$ is the unit extra production cost more than what has been planned; and $\varepsilon_{2}$ is the unit cost of handing the leftover inventory less than what has been planned. We assume $\varepsilon_{1} \leq(\mu-\tilde{\mu})^{+}$and $\varepsilon_{2} \leq(\tilde{\mu}-\mu)^{+}$, respectively.

The disruptions maybe make the cost scale bigger ( $\bar{G}(c) \geq \bar{F}(c)$, for all $c \geq 0$ ) or smaller $(\bar{G}(c) \leq \bar{F}(c)$, for all $c \geq 0)$.

Under the disruptions, we assume the optimal emergency production quantity is $\tilde{Q}^{c}$, the wholesale price is $\tilde{w}^{c}$ and the system expected profit is $\tilde{\Pi}^{c}$. We derive Theorem1.

Theorem1: If the disruptions make the retailer's cost bigger, i.e., $\tilde{\mu}>\mu, \bar{G}(c) \geq \bar{F}(c)$, for all $c \geq 0$, then $\tilde{Q}^{c} \leq Q^{c}$; otherwise if the cost is smaller, i.e., $\tilde{\mu}<\mu, \bar{G}(c) \leq \bar{F}(c)$, for all $c \geq 0$, then $\tilde{Q}^{c} \geq Q^{c}$.

There are two cases as follows.

Case 1: the cost is smaller, then $\tilde{Q}^{c} \geq Q^{c}$.

In this situation, the supply chain system's expected profit is $\tilde{\Pi}_{1}^{c}(\tilde{p})=E\left[\tilde{Q}(\tilde{p}-s-c)-\varepsilon_{1}\left(\tilde{Q}-Q^{c}\right)\right]$.

From the first optimality condition $\partial \tilde{\Pi}_{1}^{c}(\tilde{p}) / \partial \tilde{p}=0$, the optimal retail price is $\tilde{p}_{1}^{c}=\left[\alpha\left(s+\tilde{\mu}+\varepsilon_{1}\right)+b / k\right] /(\alpha-1)$. The optimal order quantity is $\tilde{Q}_{1}^{c}=(\alpha-1)^{\alpha} /\left[\alpha k\left(s+\tilde{\mu}+\varepsilon_{1}+b / k\right)\right]^{\alpha}$.

Let $A_{1}=(\alpha-1)^{\alpha-1} /\left[\alpha k\left(s+\tilde{\mu}+\varepsilon_{1}+b / k\right)\right]^{\alpha}$, $B_{1}=\varepsilon_{1}(\alpha-1)^{\alpha} /(\alpha k)^{\alpha}$, and $C_{1}=1 /\left(s+\tilde{\mu}+\varepsilon_{1}+b / k\right)^{\alpha}-1 /(s+\mu+b / k)^{\alpha}$, the supply chain system's expected profit is $\tilde{\Pi}_{1}^{c}=A_{1}\left(s+\tilde{\mu}+\alpha \varepsilon_{1}+b / k\right)-B_{1} C_{1}$.

Case 2: the retailer's cost bigger, then $\tilde{Q}^{c} \leq Q^{c}$.

In this situation, the supply chain system's expected profit is: $\tilde{\Pi}_{2}^{c}(\tilde{p})=E\left[\tilde{Q}(\tilde{p}-s-c)-\varepsilon_{2}\left(Q^{c}-\tilde{Q}\right)\right]$. From the first optimality condition $\partial \tilde{\Pi}_{2}^{c}(\tilde{p}) / \partial \tilde{p}=0$, the optimal retail price is $\tilde{p}_{2}^{c}=\left[\alpha\left(s+\tilde{\mu}-\varepsilon_{2}\right)+b / k\right] /(\alpha-1)$. The optimal order quantity is $\tilde{Q}_{2}^{c}=(\alpha-1)^{\alpha} /\left[\alpha k\left(s+\tilde{\mu}-\varepsilon_{2}+b / k\right)\right]^{\alpha}$.

Let $A_{2}=(\alpha-1)^{\alpha-1} /\left[\alpha k\left(s+\tilde{\mu}-\varepsilon_{2}+b / k\right)\right]^{\alpha}$

$B_{2}=\varepsilon_{2}(\alpha-1)^{\alpha} /(\alpha k)^{\alpha}$, and

$C_{2}=1 /(s+\mu+b / k)^{\alpha}-1 /\left(s+\tilde{\mu}-\varepsilon_{2}+b / k\right)^{\alpha}, \quad$ then the 
supply chain system's expected profit is $\tilde{\Pi}_{2}^{c}=A_{2}\left(s+\tilde{\mu}-\alpha \varepsilon_{2}+b / k\right)-B_{2} C_{2}$.

\section{NUMERICAL ANALYSIS}

In this section, we give several numerical examples to analyze the effects of optimal order quantity, and system expected profit. Let $\alpha=2, b=0.1$, $k=0.002, s=10, \varepsilon_{1}=2, \varepsilon_{2}=2$. The retailer's cost prior distribution function $F(c)$ is uniform with mean value $\mu=10$. Disruptions might cause the increase or decrease of retailer's cost. We assume the value of $\tilde{\mu}$ varies from 2 to 18 .

Fig.1 and Fig.2 illustrate the impact of varying $\tilde{\mu}$ on the optimal order quantity and the system expected profit. From those two figures, we can see that the optimal order quantity and the system expected profit is a non-linearly decreasing function of $\tilde{\mu}$. This implies that the higher $\tilde{\mu}$, the less the order quantity and the expected profits. If variation of retailer's cost is sufficiently small, the supply chain strategies are not changed.

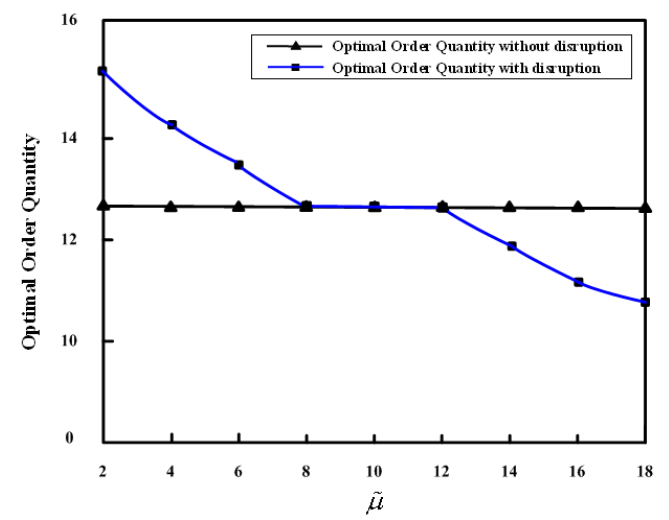

Fig.1 $\tilde{\mu}$ Versus The Optimal Order Quantity

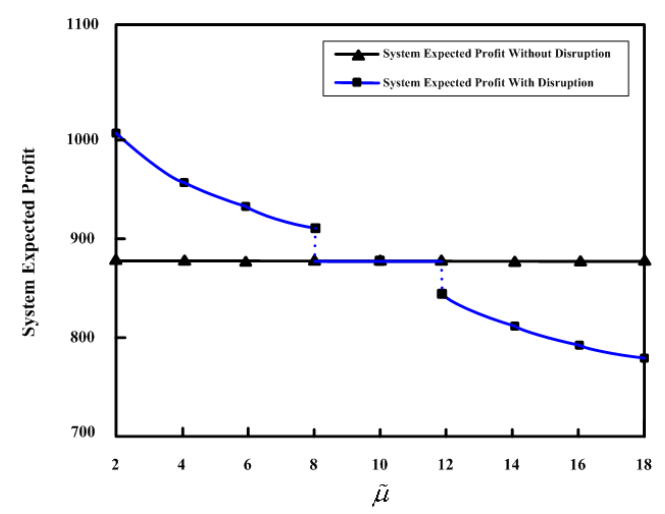

Fig.2 $\tilde{\mu}$ Versus The System Expected Profit

\section{CONCLUSION}

We have investigated supply chain contracts under asymmetric information with and without retailer's cost disruptions. Based on the analysis above, we can draw a conclusion that it is optimal for the supply chain to keep original production plan if variation of retailer's cost is sufficiently small; otherwise decisions must be adjusted.

\section{ACKNOWLEDGEMENTS}

This work was financially supported by the Education Department of Jiangsu Province University Philosophy Social Science Research Projects (NO. 2012SJD630082) and the Fundamental Research Funds for the Central Universities (NO. NR2013002).

\section{REFERENCES}

[1] F. S. Oliveira, C. Ruiz, A. J. Conejo: Contract design and supply chain coordination in the electricity industry, European Journal of Operational Research, Vol. 227 (2013), p. 527-537

[2] R. W. Seifert, R. Zequeira, S. Liao: A three-echelon supply chain with price-only contracts and sub-supply chain coordination. Int. J. Production Economics, Vol. 138 (2012), p. 345-353

[3] A. Guarnaschelli, O. Chiotti, H. Salomone. An approach based on constraint satisfaction problems to disruptive event management in supply chains, Int. J.ProductionEconomics, Vol. 144(2013), p. 223-242.

[4] A. J. Schmitt: Strategies for customer service level protection under multi-echelon supply chain disruption risk. Transportation Research Part B: Methodological, Vol. 45(8) (2011), p. 1266-1283.

[5] T. Sawik: Selection of supply portfolio under disruption risks. Omega, Vol. 39(2)(2011), p. 194-208.

[6] E. Caoa, Y. Maa, C. Wanc, M. Lai: Contracting with asymmetric cost information in a dual-channel supply chain. Operations Research Letters, Vol. 41 (2013), p. $410-414$

[7] C. L. Chu, V. J. Leon. Scalable methodology for supply chain inventory coordination with private information. European Journal of Operational Research, Vol. 195(2009), p. 262-279. 